\title{
Hollow mesoporous silica nanoparticles for intracellular delivery of fluorescent dye
}

\author{
Huichen Guo ${ }^{1}$, Haisheng Qian², Shiqi Sun ${ }^{1 *}$, Dehui Sun ${ }^{1}$, Hong Yin ${ }^{1}$, Xuepeng Cai ${ }^{1}$ Zaixin Liu', Jinyan Wu', \\ Tao Jiang ${ }^{1}$, Xiangtao Liu ${ }^{1 *}$
}

\begin{abstract}
In this study, hollow mesoporous silica nanoparticles (HMSNs) were synthesized using the sol-gel/emulsion approach and its potential application in drug delivery was assessed. The HMSNs were characterized, by transmission electron microscopy (TEM), Scanning Electron Microscopy (SEM), nitrogen adsorption/desorption and Brunauer-Emmett-Teller (BET), to have a mesoporous layer on its surface, with an average pore diameter of about 2 $\mathrm{nm}$ and a surface area of $880 \mathrm{~m}^{2} / \mathrm{g}$. Fluorescein isothiocyanate (FITC) loaded into these HMSNs was used as a model platform to assess its efficacy as a drug delivery tool. Its release kinetic study revealed a sequential release of FITC from the HMSNs for over a period of one week when soaked in inorganic solution, while a burst release kinetic of the dye was observed just within a few hours of soaking in organic solution. These FITC-loaded HMSNs was also found capable to be internalized by live human cervical cancer cells (HeLa), wherein it was quickly released into the cytoplasm within a short period of time after intracellular uptake. We envision that these HMSNs, with large pores and high efficacy to adsorb chemicals such as the fluorescent dye FITC, could serve as a delivery vehicle for controlled release of chemicals administered into live cells, opening potential to a diverse range of applications including drug storage and release as well as metabolic manipulation of cells.
\end{abstract}

\section{Background}

For many years, silica has been employed as a versatile and relatively benign material in material sciences and engineering due to the variety of available chemical and physical modifications that it offers as well as the biocompatibility feature that it display. It is thus not surprising that silica nanoparticles have recently emerged as an attractive and widely used chemical/drug delivery vehicle for basic research and clinical trials. In fact, amongst these carriers made of silica, hollow spherical silica nanoparticles with mesoporous walls stands out as a class of nanomaterials with many distinctive advantages, such as having a non-toxic nature, good surface permeability, large specific surface area, tunable pore structures, excellent physicochemical stability and chemically modifiable surfaces, all of which allow them to be potential hosts for various chemical agents/

\footnotetext{
* Correspondence: shiqisun21@hotmail.com; hnxiangtao@hotmail.com ${ }^{1}$ State Key Laboratory of Veterinary Etiological Biology and Key Laboratory of Animal Virology of Ministry of Agriculture, Lanzhou Veterinary Research Institute, Chinese Academy of Agricultural Sciences, Xujiaping 1, Lanzhou, Gansu, 730046, The People's Republic of China

Full list of author information is available at the end of the article
}

therapeutic drugs. These hollow mesoporous silica nanoparticles (HMSNs) are especially useful when used to host therapeutic compounds such as enzymes that are easily degraded in the hostile biological environment when delivered per se without encapsulation. Besides encapsulation, these therapeutic agents can also be covalently attached, or adsorbed onto such silica nanocarriers that has been pre-surface modified. Indeed, these approaches can easily overcome drug solubility and stability issues, besides having control over the rate in which the drugs are released. Thus, wide attention has been placed on the recent application of HMSNs in the field of biomedicine, including its use as novel materials in catalysis, separation, cell-labeling and capsule agent for drug delivery $[1,2]$.

To date, a number of approaches have been employed to fabricate oxide and sulfide hollow spheres $[3,4]$. Deposition of silica layer on latex or colloid templates and subsequent removal of the hard templates by calcination or corrosion has been known to be the conventional approach for preparation of HMSNs [5-7]. Solgel/emulsion method, in which the emulsion droplets are thermodynamically stable and uniform and act as
() ChemistryCentral

(c) 2011 Guo et al; licensee Chemistry Central Ltd. This is an open access article distributed under the terms of the Creative Commons Attribution License (http://creativecommons.org/licenses/by/2.0), which permits unrestricted use, distribution, and reproduction in any medium, provided the original work is properly cited. 
templates for the growth of silica, has also been developed as an alternative method for the preparation of HMSNs [8-13]. The formation of hollow silica spheres by a soft templating scheme depends on the stability of the surfactant organization, and interfacial deposition of silica to form the nanoshell. In recent years, a variety of emulsion systems, such as water-in-oil [14], oil-inwater [15-18], water-oil-water [19], oil-water-oil [20], and supercritical CO2-in-water [21,22] have all been adopted for the preparation of HMSNs. However, uniformity of the size and shell thickness of the hollow silica spheres prepared by the emulsion method are less satisfactory than those obtained from the hard template method due to the dynamic character of the emulsion droplets [23-25]. It was speculated that for oil-in-water emulsion system, introduction of another solvent that has good compatibility with both water and TEOS, for instance ethanol, might be helpful in improving stability of the oil droplets and thus monodispersity of the resulting hollow silica spheres.

In this work, we looked into the potential biomedical applications of HMSNs prepared by a sol-gel/emulsion (oil-in-water/ethanol) method. The use of ethanol is to improve the stability of the oil droplets, thus allowing the preparation of hollow silica spheres with improved size distribution. Here, the fluorescent dye FITC was chosen as a model cargo of the HMSNs for easy visualization of its release behaviour from the nanomaterial both in different solutions as well as upon uptake by cells. The possibility of combining both bioactivity and chemical agent release behaviour can make these HMSNs as an ideal tool for use in drug delivery application.

\section{Results and Discussions}

\subsection{Materials characterization}

Figure 1 shows representative TEM (Figure 1a and 1c) and SEM (Figure 1b) images of the silica spheres prepared under a certain ethanol-to-water ratios and $5 \mathrm{mM}$ CTAB concentration. These images revealed that the spheres retained their intact spherical nature even after calcination at $600^{\circ} \mathrm{C}$ for $6 \mathrm{~h}$. The spheres appear hollow in character and have an average diameter of about 200 $\mathrm{nm}$. Importantly, Figure $1 \mathrm{c}$ also revealed that the shell display a mesoporous structure that is ordered in the short range and have a shell thickness of about $20 \mathrm{~nm}$. Additionally, as these particles were dispersed in water prior to their observation under electron microscopy, the images captured also demonstrated that the nanoparticles were well dispersed in water, thus providing maximal interface to carry optimal amount of chemical agent for delivery.

The BET surface area of these HMSNs revealed a relatively high value of $880 \mathrm{~m}^{2} / \mathrm{g}$ as shown in Figure 2a, thus presenting these nanoparticles as an ideal material for hosting guest molecules of various sizes, shapes and functionalities. Their pore size distribution as depicted in Figure $2 b$ showed a wide distribution, with a sharp peak observed at around $2 \mathrm{~nm}$, thus suggesting a mesoporous reactive layer formation at the particle surface. Indeed, formation of these mesoporous structures are highly favoured as they have a high surface area and large pore, which allow chemical agents to be hosted within them in the nanoscale porosity, thereby enhancing the capacity for drug adsorption.

\subsection{FITC loading study}

The kinetics of FITC adsorption on HMSNs is as shown in Figure 3. FITC dye dissolved in acetone at a concentration of $1 \mathrm{mg} / \mathrm{mL}$ was mixed with HMSNs at different concentration for several days in order to observe the relationship between time and concentration of particles. The data seemed to suggest that there is a two-step loading pattern observe with all nanoparticle concentration, with a rapid absorbance of the dye attained in the first $24 \mathrm{~h}$ and a second relatively slow loading phase occurring in the subsequent two days. It demonstrated the absorbance of FITC to mesoporous silica nanoparticles is correlative to time point in $24 \mathrm{~h}$. The equilibrium capacity at $24 \mathrm{~h}$ was $19.95,54.68$ and $60.97 \mu \mathrm{g}$ of FITC per $\mathrm{mg}$ of nanoparticles for 1,5 and $10 \mathrm{mg} / \mathrm{mL}$ of HMSNs, respectively. This indicates that the binding of FITC is dependent on the concentration of HMSNs, as seen by the higher adsorption amount of FITC onto the HMSNs at nanoparticle concentrations of 5 and $10 \mathrm{mg} /$ $\mathrm{mL}$ than at $1 \mathrm{mg} / \mathrm{mL}$, altogether suggesting that the encapsulation of FITC is a diffusion-driven process. Taking this result into consideration, we have chosen 5 $\mathrm{mg} / \mathrm{mL}$ of nanoparticles as the concentration that is optimal for loading of FITC in all subsequent experiments.

\subsection{FITC release study}

The release studies of FITC from HMSNs $(5 \mathrm{mg} / \mathrm{mL})$ at room temperature were conducted either in inorganic solution such as PBS (pH5.2), RPMI 1640(pH7.4) or organic solution such as acetone. RPMI 1640 (pH7.4) was used to assess whether fluorescent dye could be released from HMSNs before their entry into cell. A pH value of 5.2 was chosen to mimic the physiological $\mathrm{pH}$ values inside endosomes. Figure 4 shows the cumulative release kinetics of FITC from HMSNs. The release rate of FITC from HMSNs in acetone was very rapid, showing a two-step release pattern with a rapid initial burst release and a subsequent relatively slower sustained release over five days. The initial burst observed in the first $6 \mathrm{~h}$ released around $70 \%$ of the dye, thereafter remaining almost constant throughout the next five 


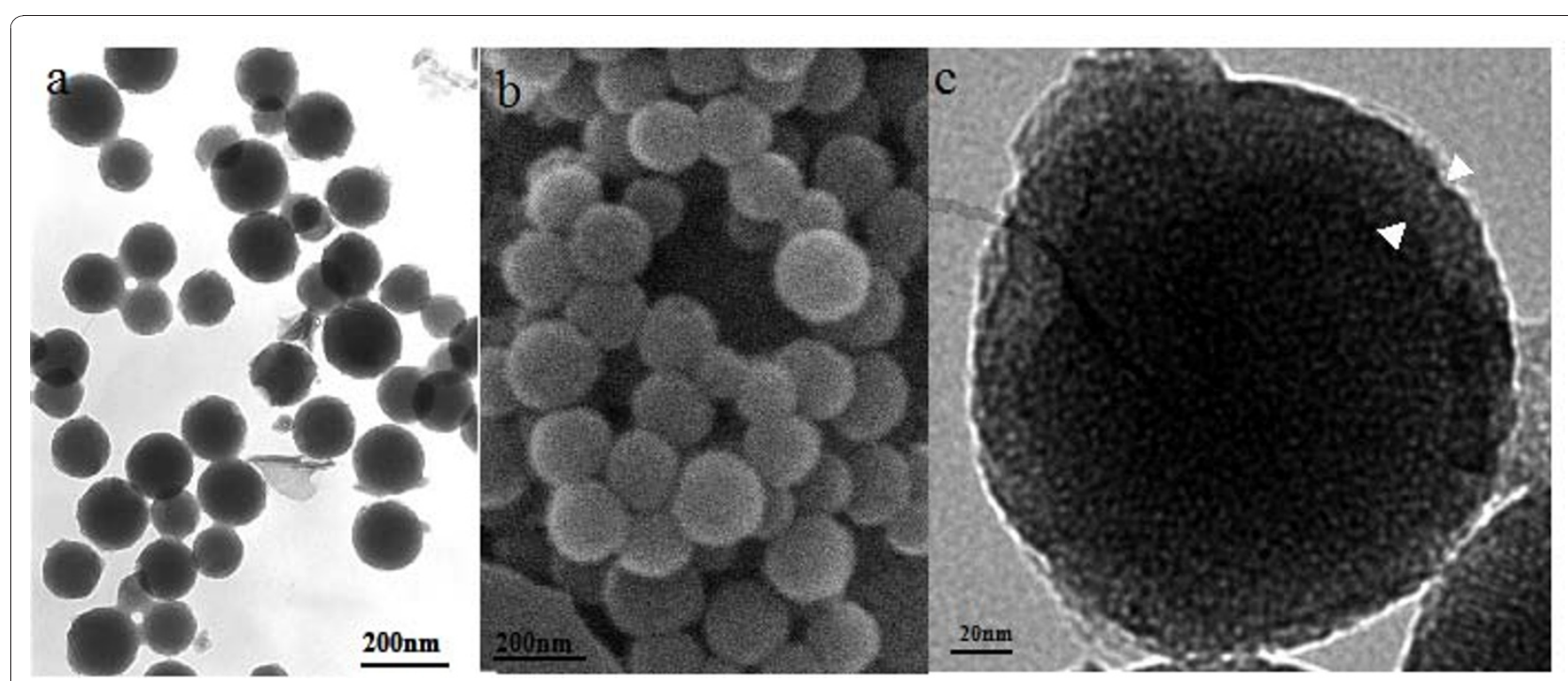

Figure 1 TEM (a) and SEM (b) images of HMSNs. TEM image of the shell of a HMSNs at high magnification is shown in (c).

days. Indeed, this is consistent with the low solubility of FITC in acetone. However, different dye release kinetics was observed from FITC-loaded HMSNs soaked in PBS (pH5.2) and cell culture medium. Initial burst released only around 33.9 and $32.6 \%$ of the dye from HMSNs soaked in PBS (pH5.2) and RPMI 1640 medium, respectively. Following this, FITC was observed to be gradually released from particles soaked in these two liquids. At the end of five days, the cumulative amount of released FITC reached a value of 68.2 and 55.6\% in PBS (pH5.2) and cell culture medium, respectively. Indeed, these were lower than that released in acetone and thus could suggest that FITC slow release from the HMSNs in cell culture medium would reduce its loss from the porous particles before the latter's entry into cells. Meanwhile, this also implies that the use of HMSNs as a drug carrier could ensure that the drug cargo is released into the cytoplasm in a slow fashion, thus appropriate for a sustained drug effect. Furthermore, recent reports on the design of functional mesoporous silica materials that have their pore surface decorated with organic or inorganic moieties to serve as gating devices that can regulate the release of guest molecules under the control of several different external stimuli, such as chemicals [26-29], temperature [30], redox reactions [31,32], and photoirradiation [33,34], have highlighted the potential of utilizing such kind of nanodevice for diverse controlled release applications. Indeed, this could be incorporated in our future work for controlled release of our HMSNs by utilizing such different moieties.
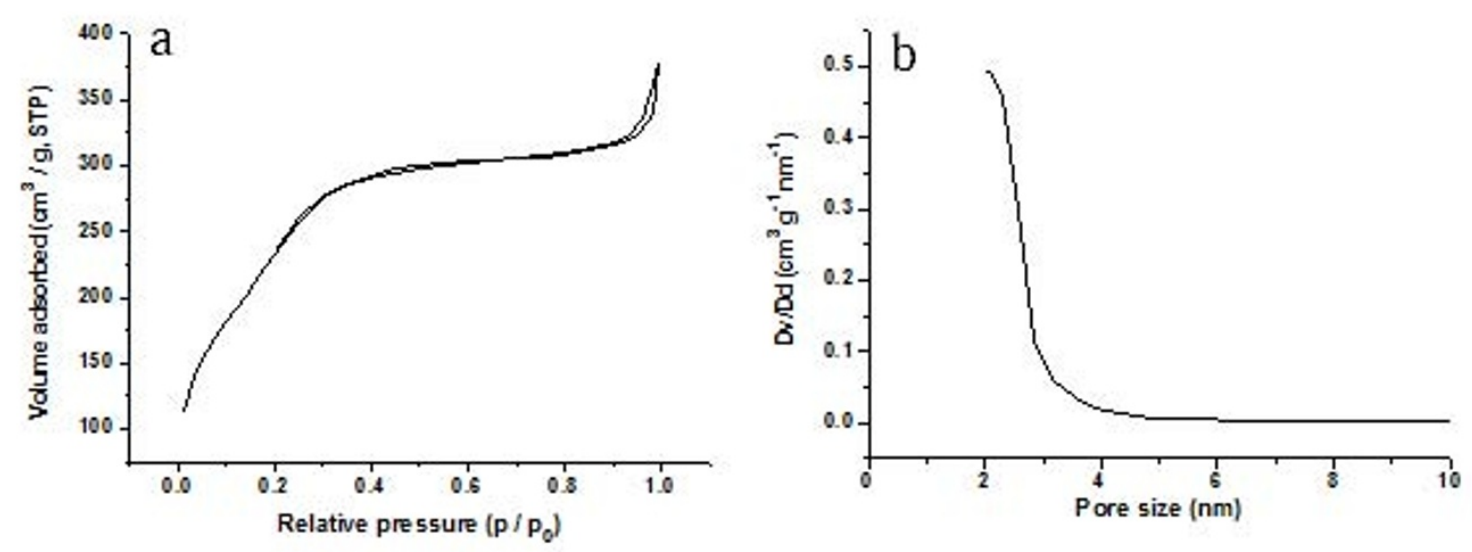

Figure 2 N2 adsorption/desorption isotherms (a) and BET pore size distribution plots (b) of the calcined HMSNs by a sol-gel/emulsion method. 


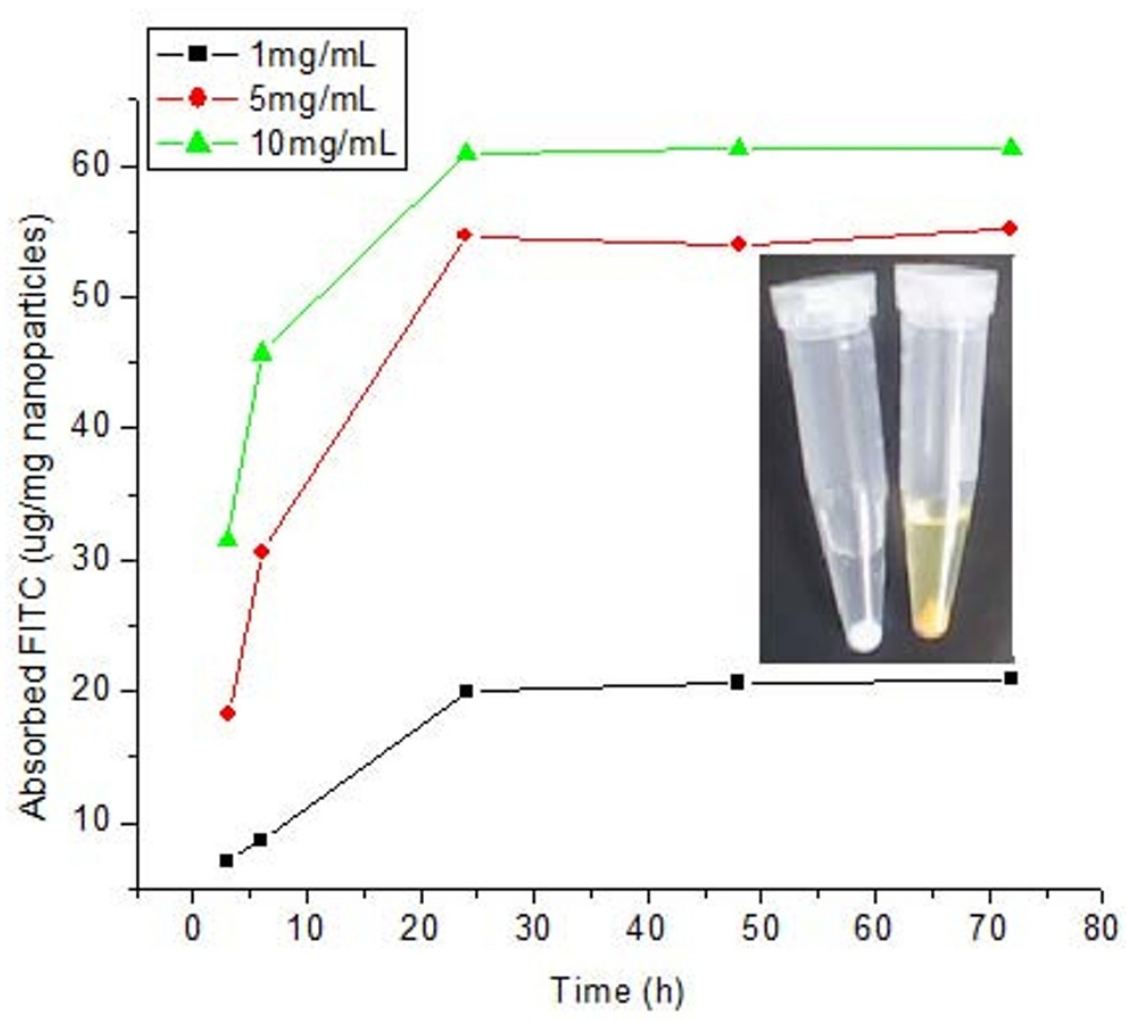

Figure 3 Kinetics study of FITC adsorption on HMSNs at different nanoparticle concentration. Insert displays a photograph of HMSNS before (white color) and after (yellow color) soaking in FITC-acetone solution.

\subsection{Biocompatibility test in vitro}

Non-Radioactive Cell Proliferation Assay [(3- $(4,5-$ dimethylthiazol-2yl)-5-(3-carboxymethoxyphenyl)-2-(4sulfophenyl)-2H-tetra zolium, inner sath; MTS) was used to determine the proliferation and viability of cells that has been treated with different concentrations of the nanoparticles. Figure 5 shows the densities of cells cultured with different concentrations of HMSNs for 24 and $48 \mathrm{~h}$. Even though cell viability was observed to decrease as a function of concentration and time, it was noted that none of the cell samples reached below $70 \%$ of viability. As such, this indicates that even at a high concentration of $2000 \mu \mathrm{g} / \mathrm{mL}$, the nanoparticles exert no significant cytotoxic effect on the cells and thus presents as a safe and ideal delivery vehicle or chemical/drugs into cells.

\subsection{Imaging of FITC-HMSNs labelled cells}

The uptake of FITC- mesoporous silica nanoparticles by HeLa cells was confirmed by confocal fluorescence microscopy. FITC-loaded HMSNs were passively loaded into Hela cells by incubating them with $100 \mu \mathrm{g} / \mathrm{mL}$ of the nanoparticles. Figure 6 shows that the HMSNs were endocytosed into cells as early as $3 \mathrm{~h}$ after exposure to the nanoparticles and a progressive increase in fluorescence emitted by nanoparticle-labelled cells was observed with increased in time point. The fact that nanoparticles uptake by Hela cells was significantly higher at $6 \mathrm{~h}$ as compared to $3 \mathrm{~h}$ of incubation highlights the time-dependent accumulation of these nanoparticles within the cells. Recently, it was demonstrated that particle size determines the mechanism and rate of intracellular uptake as well as ability of the particle to permeate through tissue $[35,36]$. Indeed, the size of a particle can affect the efficiency and pathway of its cellular uptake by influencing their adhesion and interaction with cells [37]. For biomedical applications, the control of pore size, morphology, and particle size of ordered mesoporous silica is thus important. Compared to bulky mesoporous silica materials, which have sizes above a micrometer, nanometer-sized mesoporous silica particles (30 to $500 \mathrm{~nm}$ ) are better transporters since they have a fast mass transport, effective adhesion to substrates, and good suspension in solution. In this report, the size of mesoporous silica nanoparticles of about $200 \mathrm{~nm}$ in diameter, is therefore ideal for cellular uptake. It is expected that the size effect on cell uptake would lead to size-dependent biochemical 


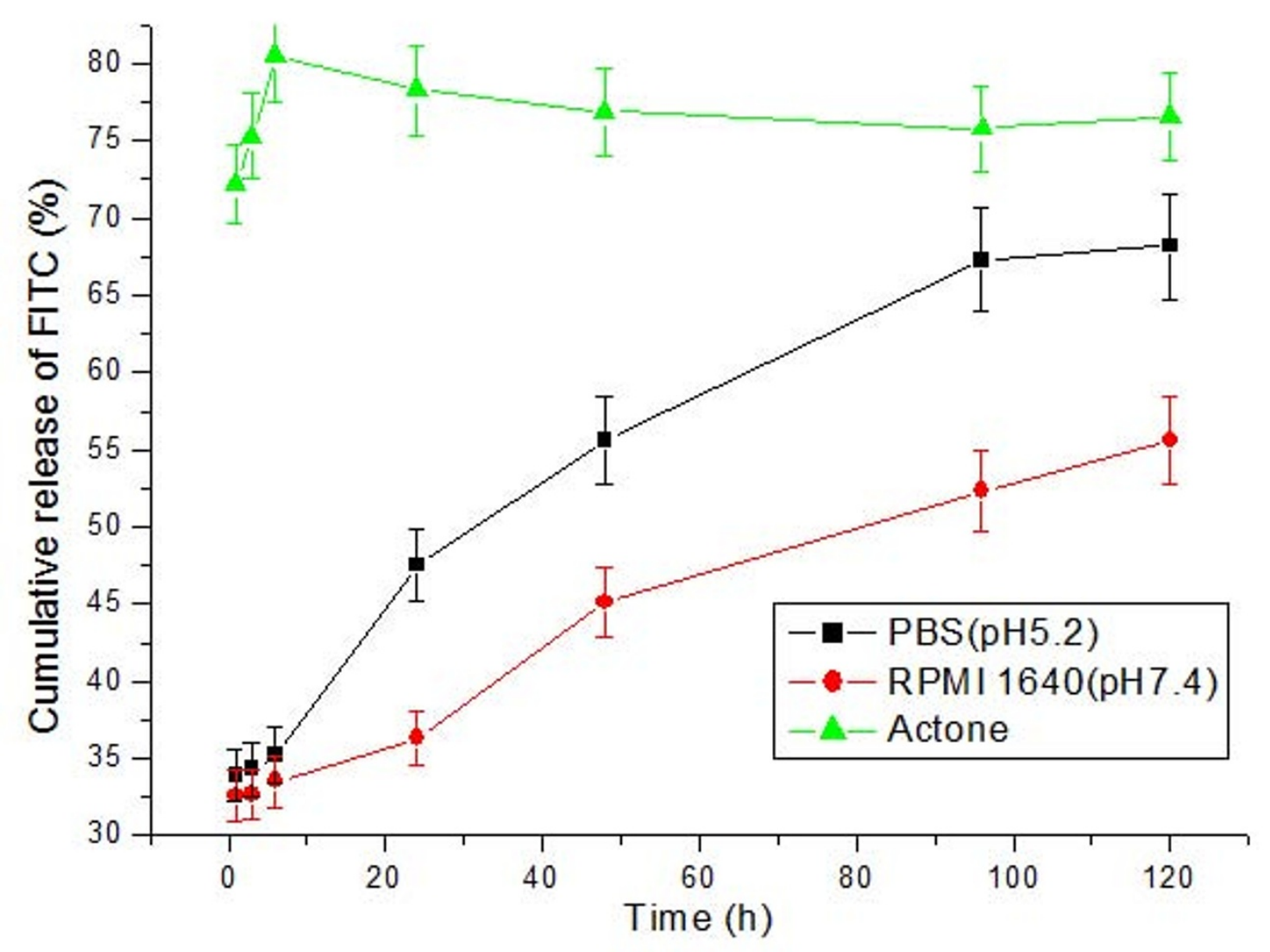

Figure 4 Cumulative release kinetics of FITC from HMSNs in different solutions.

responses [38]. The detailed downstream cell response, however, needs further in depth study.

\subsection{Endosomeal release of FITC-loaded HMSNs}

To determine whether the FITC-loaded HMSNs could escape the endosomal entrapment, we stained the endosomes with a red fluorescent antibody and monitored the distribution of FITC loaded HMSNs in HeLa cells by confocal fluorescence micrographs. The yellow fluorescent spots (indicative of a merged between the red and green spots) observed in Figure 7 represent the FITC-loaded HMSNs that has remained trapped in endosomes, while those that were released from the endosomes exhibited a green color. This clearly shows that the FITC-HMSNs could escape the endosomal compartment efficiently within a few hours of endocytosis. Interestingly, several red spots with no overlapping fluorescence from the green FITC-HMSNs were also observed in the cell. This could either be endosomes that have not harbour any FITC-mesoporous silica nanoparticles in them or those that have already emptied their nanoparticulate contents into the cytoplasm.
To further confirm release of HMSNs from the endosomes, cells were incubated with FITC-loaded HMSNs and their intracellular localization was investigated by TEM at $3 \mathrm{~h}$ time point. The results as shown in Figure 8c demonstrate that HMSNs adhered to the plasma membrane were subsequently internalized by a vesicle-mediated endocytosis process. This is evident by appearance of some cytoplasmic vacuoles harbouring non-clustered nanoparticles in Figure 8c-d. Additionally, nanoparticles located outside the endosomes were also observed as shown in Figure 8d., which is consistent with the previous result obtained by confocal fluorescence microscopy. This further emphasizes the fact that HMSNs are able to escape the endolysosomal entrapment in just a short period of time after their intracellular uptake. At all phases of HMSNs interaction with cells, the particles predominantly maintained their uniformity in shape and showed absence of aggregate formation. Therefore, these particles could serve not only as vehicles for introducing drugs/chemicals into cells, but are also capable of releasing their cargo into the cytoplasm. Nonetheless, the detailed mechanism on the 


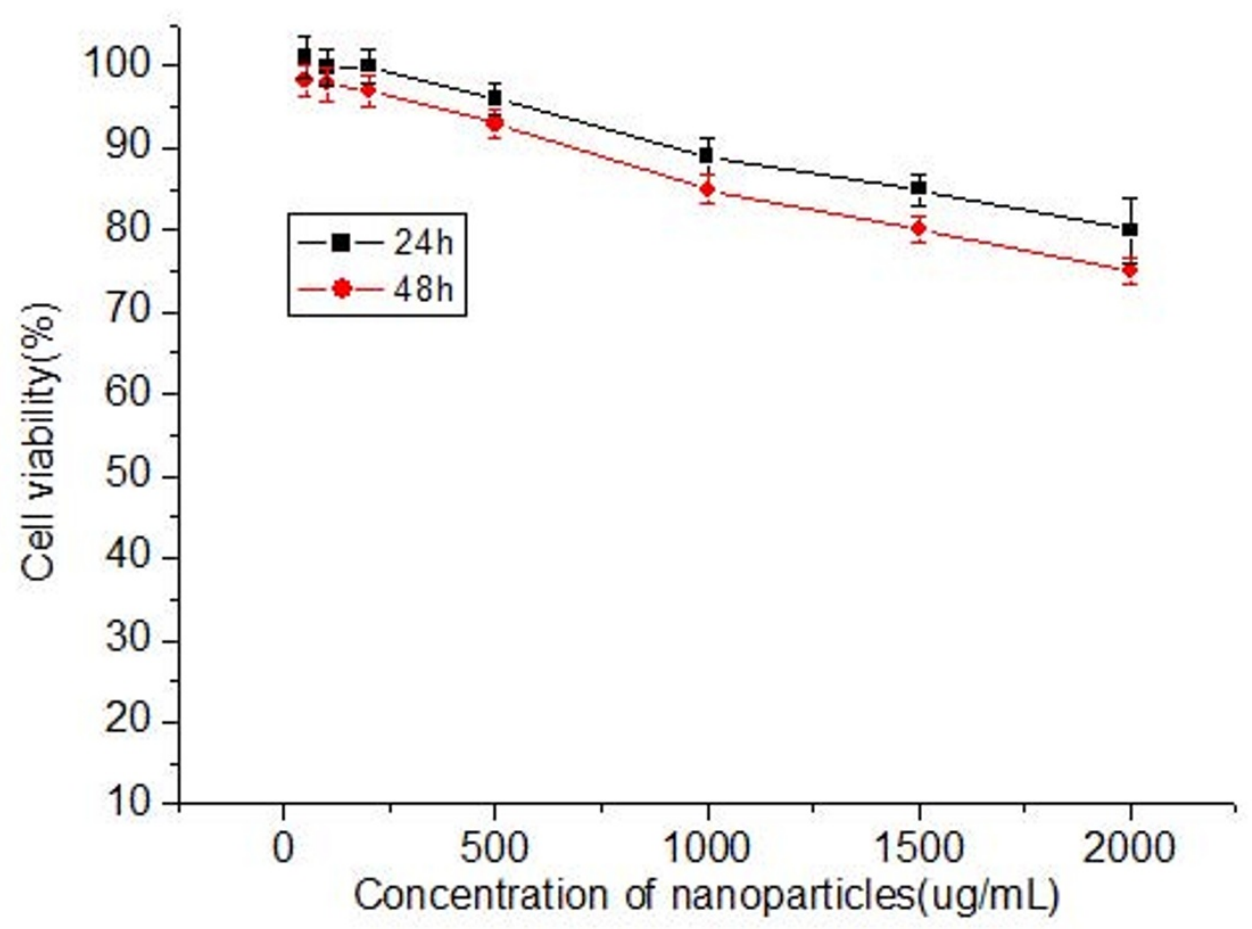

Figure 5 Influence of different concentrations of HMSNs on the cell viability of Hela cells.

escape of these particles from the endosomes is not yet clearly elucidated and warrants further research works.

\section{Conclusions}

Hollow mesoporous silica spheres were prepared by a sol-gel/emulsion (oil-in-water/ethanol) method wherein hydrated silica gel was formed on the surface of HMSNs. This surface layer had a mesoporous structure, with a pore diameter of about $2 \mathrm{~nm}$. FITC adsorption studies revealed that HMSNs had a high adsorption capacity for FITC at high concentration of the nanoparticles. Their release kinetics showed that FITC adsorbed onto these HMSNs could be released continually over one week in inorganic solution, whereas a burst release kinetics of the fluorescent dye was observed just within a few hours in organic solution. The synthesized nanoparticles also showed good biocompatibility with Hela cells wherein they show ability to be released from the endosomal compartment after intracellular uptake by endocytosis. In summary, this work demonstrated the potential of producing HMSNs as a carrier for protein/drug delivery for imaging and other biomedical applications.

\section{Experimental}

\subsection{Materials}

Tetraethyl orthosilicate (TEOS) (A.R.), anhydrous ethanol, cetyltrimethylammonium bromide (CTAB) (A.R.) and ammonia (25\%) were purchased from Tianjin
Chemical Corporation (Tianjin, China). Deionized water was prepared with an ion exchange System

\subsection{Synthesis of hollow mesoporous silica spheres}

HMSNs were synthesized by the ammonia-catalyzed hydrolysis and condensation of TEOS in mixed ethanolwater solvents, with $\mathrm{CTAB}$ used as a surfactant. TEOS and CTAB were first dispersed in $13 \mathrm{~mL}$ 95\% ethanol and 27.5 $\mathrm{mL}$ water. $25 \%$ ammonia solution was then added after diluting it with ethanol, and this was kept stirring at 700 $\mathrm{rpm}$ for $3 \mathrm{~h}$. When the reaction was completed, the resultant products were collected by centrifugation and washed with water. Finally the stable spheres with hollow structure were obtained by calcinations at $200^{\circ} \mathrm{C}$ with an increment rate of $5^{\circ} \mathrm{C} / \mathrm{min}$ for $6 \mathrm{~h}$ and then at $600^{\circ} \mathrm{C}$ for $6 \mathrm{~h}$.

\subsection{Characterization of hollow mesoporous silica spheres}

Transmission Electron Microscopy (TEM) images were recorded on a JEOL 2010 transmission electron microscope operated at an acceleration voltage of $200 \mathrm{kV}$. Samples for TEM measurements were prepared by dipping a drop of the colloidal solution onto Formvar coated copper grids. Scanning Electron Microscopy (SEM) images were taken on a Shimadzu SSX-550 field emission scanning electron microscope at $15.0 \mathrm{kV}$. N2 adsorption-desorption isotherm measurements were carried out on a Quantachrome Autosorb-1 pore analyzer at $77 \mathrm{~K}$ under a continuous adsorption condition. 


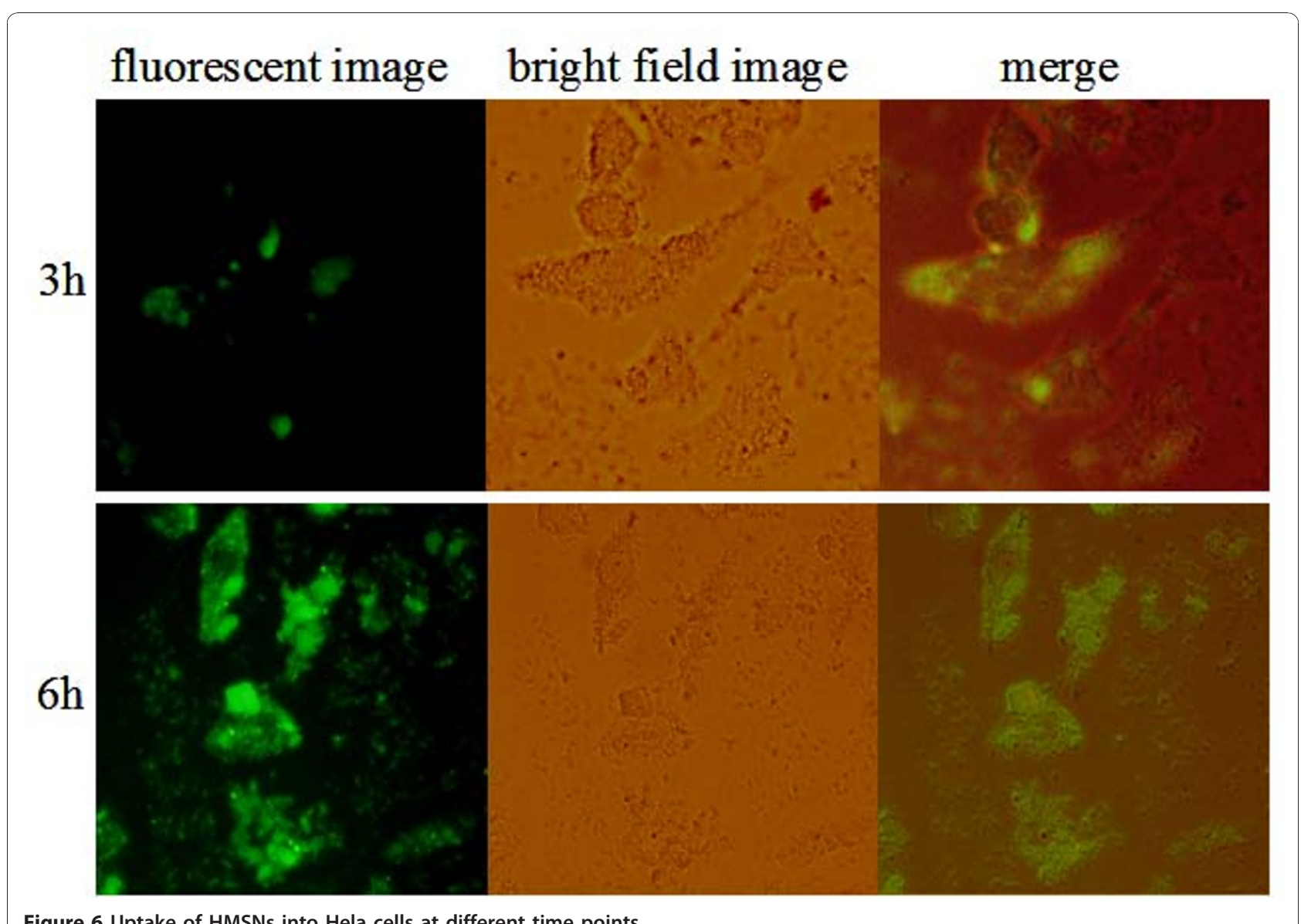

Figure 6 Uptake of HMSNs into Hela cells at different time points.

Samples were degassed at $200^{\circ} \mathrm{C}$ for $6 \mathrm{~h}$ before taking the measurements. Brunauer-Emmett- Teller (BET) analyses was used to determine the surface areas and pore sizes of the products.

\subsection{Loading of FITC into hollow mesoporous silica spheres}

FITC was used as a model drug and the effect of different particle concentration on drug loading was studied. Different concentrations of HMSNs (1, 5 and $10 \mathrm{mg} /$ $\mathrm{mL}$ ) were mixed with $1 \mathrm{~mL}$ of $1 \mathrm{mg} / \mathrm{mL}$ FITC in acetone solution. The mixture was then incubated for different time duration at ambient temperature, followed by centrifugation at $5000 \mathrm{rpm}$ for $5 \mathrm{~min}$. FITC was quantified, using a UV-vis spectrophotometer, according to its absorbance peak at $488 \mathrm{~nm}$. Raw data were converted to concentration $(\mu \mathrm{g} / \mathrm{mg})$ of FITC using the standard curves obtained at different time point.

\subsection{Release profiles of FITC from hollow mesoporous silica spheres}

The FITC-loaded HMSNs, obtained as described in section 2.4, were mixed with $1 \mathrm{~mL}$ of RPMI 1640 medium
(pH 7.4), acetone or PBS ( $\mathrm{pH}$ 5.2) and left incubated at ambient temperature under gentle shaking. A $100 \mu \mathrm{L}$ aliquot of supernatant was removed at a given time after centrifugation at $5000 \mathrm{rpm}$ for $5 \mathrm{~min}$ and this was supplemented with $100 \mu \mathrm{L}$ of fresh PBS, acetone or RPMI 1640 medium, respectively. The absorbance of FITC at $488 \mathrm{~nm}$ was recorded, and the cumulative release of FITC was calculated. One-way ANOVA and Duncan's multiple-range test for post hoc comparisons were carried out, and $\alpha=0.05$ significant level was used.

\subsection{In vitro cellular viability tests}

Hela cells were cultured in RPMI-1640 medium (Gibco, Life Technologies, Vienna,Austria) supplemented with $10 \%$ fetal bovine serum (Gibco, Life Technologies, Vienna, Austria), $100 \mathrm{U} / \mathrm{mL}$ penicillin and $0.1 \mathrm{mg} / \mathrm{mL}$ streptomycin (Geneticin, GibcoBRL life Technology, Inc). Cells were maintained at $37{ }^{\circ} \mathrm{C}$ in a humidified $5 \%$ $\mathrm{CO} 2 \mathrm{atmosphere.} \mathrm{For} \mathrm{MTS} \mathrm{assay,} \mathrm{Hela} \mathrm{cells} \mathrm{were} \mathrm{col-}$ lected and diluted to a density of $1 \times 10^{5}$ cells $/ \mathrm{mL}$ in complete medium, and then seeded onto 96-well plates $(100 \mu \mathrm{L} /$ well). After an overnight culture of $24 \mathrm{~h}$, nanoparticles of different concentrations $(50,100,200,500$, 


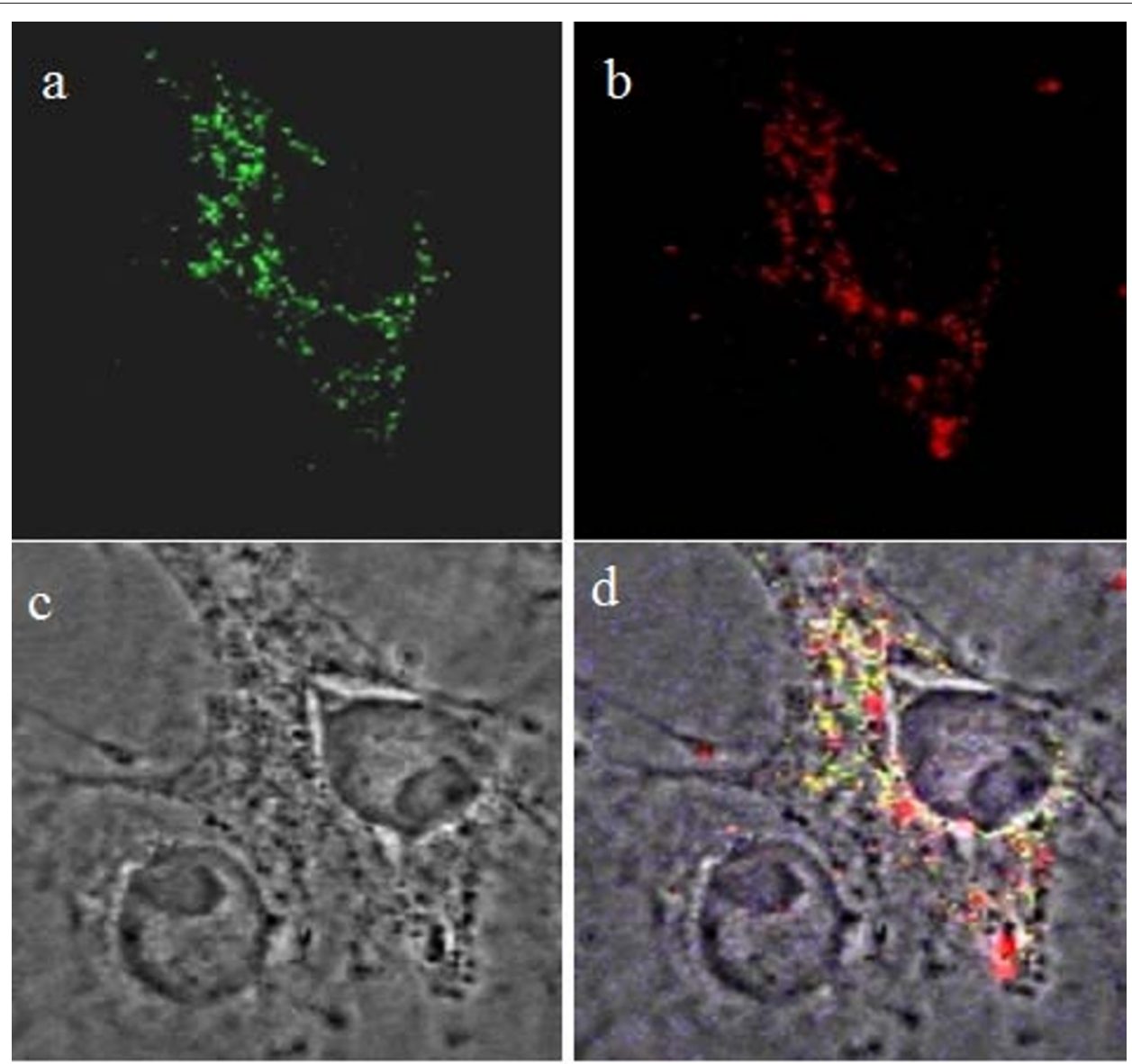

Figure 7 Cellular release of FITC-loaded HMSNs from endosomes in Hela cells. (a) FITC-loaded HMSNs; (b) endosomes; (c) bright field image; (d) merge.

$1000,1500,2000 \mathrm{ug} / \mathrm{mL}$ ) were added to the cells and then incubated for another 24 or $48 \mathrm{~h}$ at $37{ }^{\circ} \mathrm{C}$. Its viability was assessed using the Cell Titer 96 aqueous one solution assay (Promega, Madison, WI). The reagent contains tetrazolium compound [3-(4,5-dimethylthiazol2yl)-5-(3-carboxymethoxyphenyl)-2-(4-sulfophenyl)-2Htetra zolium, inner sath; MTS]. The relative cell viability (\%) related to control wells containing cell culture medium without nanocrystals was calculated by [A]expt/[A] control $\times 100$, where $[\mathrm{A}]$ expt is the absorbance of the test sample and $[\mathrm{A}]$ control is the absorbance of control sample.

\subsection{Cell imaging}

Hela cells were grown on a glass cover slide for $24 \mathrm{~h}$. The HMSNs $(100 \mu \mathrm{g} / \mathrm{mL})$

Loaded with FITC were added and the cells were incubated for varying times ( 3 and $6 \mathrm{~h}$ ). The cells were imaged in bright field and under UV infrared excitation using a Nikon confocal microscope.

\subsection{Intra cellular release study of FITC-loaded nanoparticles}

Hela cells were grown overnight on glass-bottom culture dishes (MattekTM), and then incubated with culture medium containing $100 \mu \mathrm{g} / \mathrm{mL}$ of FITC-loaded HMSNs for $3 \mathrm{~h}$ at $37^{\circ} \mathrm{C}$. Release of FITC-loaded nanoparticles from the endosomes were imaged by staining the endosomes with a rabbit polyclonal antibody to EEA1 (early endosome marker, Molecular Probe) and visualized with Alexa Fluor 568 goat anti-rabbit secondary antibody (Molecular Probe). The stained cells were then imaged using a Zeiss LSM 510 laser-scanning microscope (Thornwood, New York). Images were captured using the Evolution MP Cooled Camera Kit (Corporate Headquarters, MD, USA). To acquire TEM images of the cells, they were fixed with $2.5 \%$ glutaraldehyde (Electron Microscopy Sciences) in $0.1 \mathrm{M}$ sodium cacodylate (Ted PellaTM) buffer ( $\mathrm{pH} \mathrm{7.3)} \mathrm{for} 20 \mathrm{~min}$ at room temperature. This was followed by washing with $0.1 \%$ cacodylate buffer, and postfixing with $1 \%$ osmium tetroxide 


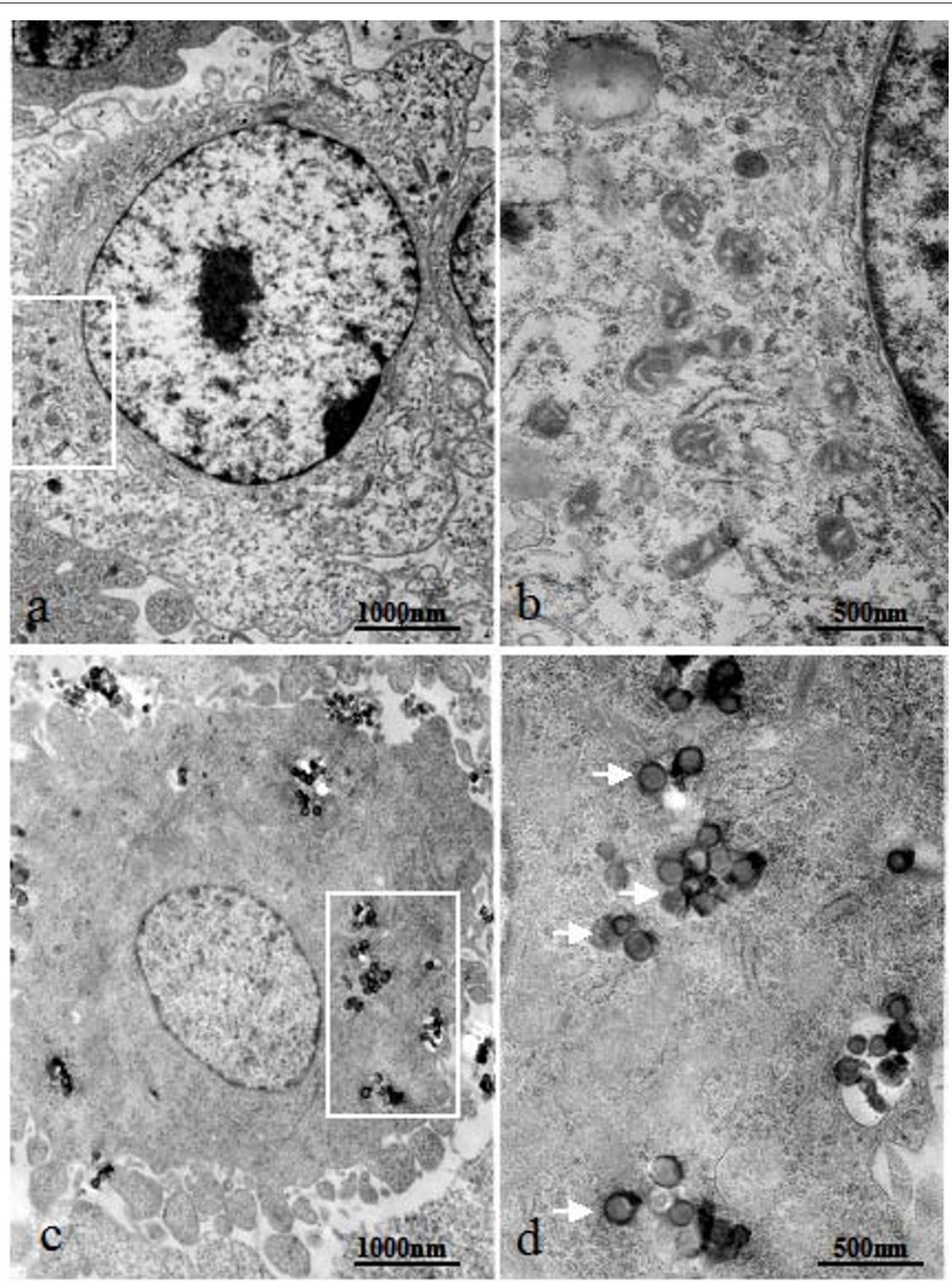

Figure 8 Transmission electron micrographs of Hela cells treated for $\mathbf{3} \mathbf{h}$ with FITC-loaded HMSNs. (a) Untreated cells; (b) Magnified regions of endosomes in (a) at higher power; (c) Cells treated with FITC-loaded nanoparticles; (d) Magnified regions of endosomes in (b) at higher power; White arrows indicate particles that has been released from the endosomes.

(Electron Microscopy Sciences) solution for $30 \mathrm{~min}$. Subsequently, the cells were counterstained with $4 \%$ uranyl acetate (Electron Microscopy Sciences) for 30 min, washed with distilled water, dehydrated in 100\% ethanol, and embedded in DurcupanTM ACM resin (FlukaTM).

\section{Abbreviations}

FITC: Fluorescein isothiocyanate; HMSNs: Hollow mesoporous silica nanoparticles; TEM: Transmission electron microscopy; SEM: Scanning Electron Microscopy; TEOS: Tetraethyl orthosilicate; CTAB:

Cetyltrimethylammonium bromide; BET: Brunauer-Emmett-Teller; MTS: 3-(4,5-
dimethylthiazol-2yl)-5-(3-carboxymethoxyphenyl)-2-(4-sulfophenyl)-2H-tetra zolium, inner sath; EEA1: early endosome marker;

\section{Acknowledgements}

This work was supported by the ministry of science and technology of China (No.2008FY130100) and science and technology committee of Gansu (No.1002NKDA037).

\section{Author details}

${ }^{1}$ State Key Laboratory of Veterinary Etiological Biology and Key Laboratory of Animal Virology of Ministry of Agriculture, Lanzhou Veterinary Research Institute, Chinese Academy of Agricultural Sciences, Xujiaping 1, Lanzhou, Gansu, 730046, The People's Republic of China. ${ }^{2}$ Department of Chemistry, Zhejiang Normal University, Jinhua, 321004, The People's Republic of China. 


\section{Authors' contributions}

HG has made substantial contributions to conception and design, acquisition of data, analysis and interpretation of data, involved in drafting the manuscript and revising it critically for important intellectual content. HQ, DS, JW, JT involved in acquisition of data, analysis and interpretation of data. ZL, HY, XC, XL, SS revised it critically for important intellectual content; and have given final approval of the version to be published.

\section{Competing interests}

The authors declare that they have no competing interests.

Received: 4 November 2010 Accepted: 5 January 2011

Published: 5 January 2011

\section{References}

1. Yang J, Lee J, Kang J, Lee K, Suh JS, Yoon HG, Huh YM, Haam S: Hollow silica nanocontainers as drug delivery vehicles. Langmuir 2008, 24(7):3417-3421.

2. Song XF, Gao L: Fabrication of hollow hybrid microspheres coated with silica/titania via sol-gel process and enhanced photocatalytic activities. J Phy Chem C 2007, 111:8180-8187.

3. Le Y, Chen JF, Wang JX, Shao L, Wang WC: A novel pathway for synthesis of silica hollow spheres with mesostructured walls. Materials Letters 2004, 58(15):2105-2108.

4. Caruso F, Caruso AC, ohwald HM: Nanoengineering of inorganic and hybrid hollow spheres by colloidal templating. Science 1998, 282:1111-1114.

5. Jiang $Y$, Ding $X$, Zhao J, Hari B, Zhao X, Tian Y, Yu K, Sheng Y, Guo Y, Wang Z: A facile route to synthesis of hollow $\mathrm{SiO} 2 / \mathrm{Al} 2 \mathrm{O} 3$ spheres with uniform mesopores in the shell wall. Materials Letters 2005, 59(23):2893-2897.

6. Song L, Ge X, Wang M, Zhang Z: Direct preparation of silica hollow spheres in a water in oil emulsion system: The effect of $\mathrm{pH}$ and viscosity. Journal of Non-Crystalline Solids 2006, 352(21-22):2230-2235.

7. Singh RK, Garg A, Bandyopadhyaya R, Mishra BK: Density fractionated hollow silica microspheres with high-yield by non-polymeric sol-gel/ emulsion route. Colloids and Surfaces A: Physicochemical and Engineering Aspects 2007, 310(1-3):39-45.

8. lida M, Sasaki T, Watanabe M: Titanium dioxide hollow microspheres with an extremely thin shell. Chem Mater 1998, 10:3780-3786.

9. Yang Y, Chu Y, Zhang Y, Yang F, Liu J: Polystyrene-ZnO core-shell microspheres and hollow $\mathrm{ZnO}$ structures synthesized with the sulfonated polystyrene templates. Journal of Solid State Chemistry 2006, 179(2):470-475.

10. Agrawal M, Pich A, Gupta S, Zafeiropoulos NE, Simon P, Stamm M: Synthesis of novel tantalum oxide sub-micrometer hollow spheres with tailored shell thickness. Langmuir 2008, 24:1013-1018.

11. Song C, Gu G, Lin Y, Wang H, Guo Y, Fu X, Hu Z: Preparation and characterization of CdS hollow spheres. Materials Research Bulletin 2003, 38(5):917-924

12. Cong $\mathrm{YH}$, Wang $\mathrm{GL}$, Xiong $\mathrm{MH}$, Huang $\mathrm{YJ}$, Hong $\mathrm{ZF}$, Wang $\mathrm{DL}$, et al: A facile interfacial reaction route to prepare magnetic hollow spheres with tunable shell thickness. Langmuir 2008, 24:6624-6629.

13. Wang $D$, Song $C$, Lin Y, Hu Z: Preparation and characterization of TiO2 hollow spheres. Materials Letters 2006, 60(1):77-80

14. Li WJ, Sha XX, Dong WJ, Wang ZC: Synthesis of stable hollow silica microspheres with mesoporous shell in nonionic W/O emulsion. Chem Commun 2002, 20: 2434-2435.

15. Li YS, Shi JL, Hua ZL, Cheng HR, Ruan ML, Yan DS: Hollow Spheres of Mesoporous Aluminosilicate with a Three-Dimensional Pore Network and Extraordinarily High Hydrothermal Stability. Nano lett 2003, 3(5):609-612.

16. Giri S, Trewyn BG, Stellmaker MP, Lin VS: Stimuli-responsive controlledrelease delivery system based on mesoporous silica nanorods capped with magnetic nanoparticles. Angew Chem Int Ed Engl 2005, 44(32):5038-5044.

17. Christabel EF, Deepa K, Stephen M: Interfacial synthesis of hollow microspheres of mesostructured silica. Chem Commun 2001, 19: 2028-2029.
18. Zhang HJ, Wu J, Zhou LP, Zhang DY, Qi LM: Facile Synthesis of Monodisperse Microspheres and Gigantic Hollow Shells of Mesoporous Silica in Mixed Water-Ethanol Solvents. Langmuir 2007, 23(3):1107-1113.

19. Masahiro F, Kumi S, Yuko T, Yoshiko N: Preparation and Formation Mechanism of Silica Microcapsules (Hollow Sphere) by Water/Oil/Water Interfacial Reaction. Chem Mater 2004, 16(25):5420-5426.

20. Li WJ, Coppens MO: Synthesis and Characterization of Stable Hollow TiSilica Microspheres with a Mesoporous Shell. Chem Mater 2005, 17(9):2241-2246.

21. Wang JW, Xia YD, Wang WX, Robert M, Martyn P: Synthesis of siliceous hollow spheres with large mesopore wall structure by supercritical $\mathrm{CO}_{2}-$ in-water interface templating. Chem Commun 2005, , 2: 210-212.

22. Wang JW, Xia YD, Wang WX, Martyn P, Robert M: Synthesis of mesoporous silica hollow spheres in supercritical CO2/water systems. J Mater Chem 2006, 16:1751-1756.

23. Zhu YF, Shi JL, Li YS, Chen HR, Shen WH, Dong XP: Storage and release of ibuprofen drug molecules in hollow mesoporous silica spheres with modified pore surface. Microporous and Mesoporous Materials 2005, 85(12):75-81.

24. Chen JF, Ding HM, Wang JX, Shao L: Preparation and characterization of porous hollow silica nanoparticles for drug delivery application. Biomaterials 2004, 25(4):723-727.

25. Shi JL, Hua ZL, Zhang LX: Nanocomposites from ordered mesoporous materials. J Mater Chem 2004, 14(5):795-806

26. Giri S, Trewyn BG, Stellmaker MP, Lin VS: Stimuli-responsive controlledrelease delivery system based on mesoporous silica nanorods capped with magnetic nanoparticles. Angew Chem Int Ed Engl 2005, 44(32):5038-5044.

27. Lai CY, Trewyn BG, Jeftinija DM, Jeftinija K, Xu S, Jeftinija S, Lin VS: A mesoporous silica nanosphere-based carrier system with chemically removable CdS nanoparticle caps for stimuli-responsive controlled release of neurotransmitters and drug molecules. J Am Chem Soc 2003, 125(15):4451-4459

28. Radu DR, Lai CY, Jeftinija K, Rowe EW, Jeftinija S, Lin VS: A polyamidoamine dendrimer-capped mesoporous silica nanosphere-based gene transfection reagent. J Am Chem Soc 2004, 126(41):13216-13217.

29. Nguyen TD, Leung KC, Liong M, Pentecost CD, Stoddart JF, Zink Jl: Construction of a pH-driven supramolecular nanovalve. Org Lett 2006, 8(15):3363-3366

30. Fu Q, Rao GVR, Ista LK, Wu Y, Andrzejewski BP, Sklar LA, Ward TL, Lopez GP: Control of Molecular Transport Through Stimuli-Responsive Ordered Mesoporous Materials. Advanced Materials 2003, 15(15):1262-1266.

31. Hernandez R, Tseng HR, Wong JW, Stoddart JF, Zink Jl: An operational supramolecular nanovalve. J Am Chem Soc 2004, 126(11):3370-3371.

32. Nguyen TD, Tseng HR, Celestre PC, Flood AH, Liu Y, Stoddart JF, Zink Jl: A reversible molecular valve. Proc Natl Acad Sci USA 2005, 102(29):10029-10034.

33. Mal NK, Bhaumik A, Kumar P, Fujiwara M: Microporous niobium phosphates and catalytic properties prepared by a supramolecular templating mechanism. Chem Commun (Camb) 2003, , 7: 872-873.

34. Mal NK, Fujiwara M, Tanaka Y: Photocontrolled reversible release of guest molecules from coumarin-modified mesoporous silica. Nature 2003, 421(6921):350-353

35. Tallury P, Payton K, Santra S: Silica-based multimodal/multifunctional nanoparticles for bioimaging and biosensing applications. Nanomedicine (Lond) 2008, 3(4):579-592.

36. Smith AM, Duan H, Mohs AM, Nie S: Bioconjugated quantum dots for in vivo molecular and cellular imaging. Adv Drug Deliv Rev 2008, 60(11):1226-1240.

37. Lee KD, Nir S, Papahadjopoulos D: Quantitative analysis of liposome-cell interactions in vitro: rate constants of binding and endocytosis with suspension and adherent $\mathrm{J774}$ cells and human monocytes. Biochemistry 1993, 32(3):889-899.

38. Zhang S, Li J, Lykotrafitis G, Bao G, Suresh S: Size-Dependent Endocytosis of Nanoparticles. Adv Mater Deerfield 2009, 21:419-424.

doi:10.1186/1752-153X-5-1

Cite this article as: Guo et al: Hollow mesoporous silica nanoparticles for intracellular delivery of fluorescent dye. Chemistry Central Journal 2011 5:1 